\title{
Thermally Responsive Composite Hydrogel via Self-Assembly for Smart Window Applications
}

\author{
Yibo Feng, ${ }^{1}$ Qingquan Zhang, ${ }^{2}$ Hui Li, ${ }^{1}$ Yongjin Chen, ${ }^{1}$ and Cong Wang ${ }^{1}$ \\ ${ }^{1}$ Institute of Microstructure and Property of Advanced Materials, Beijing University of Technology, Beijing 100124, China \\ ${ }^{2}$ Department of Physics, Hong Kong University of Science and Technology, Clear Water Bay, Kowloon, Hong Kong \\ Correspondence should be addressed to Cong Wang; smartswang@163.com
}

Received 21 January 2016; Revised 13 July 2016; Accepted 21 July 2016

Academic Editor: Jeffrey Elam

Copyright (c) 2016 Yibo Feng et al. This is an open access article distributed under the Creative Commons Attribution License, which permits unrestricted use, distribution, and reproduction in any medium, provided the original work is properly cited.

\begin{abstract}
A novel thermally responsive hydrogel (TRH) has been demonstrated by confining poly(ethylene oxide), poly(propylene oxide), and poly(ethylene oxide) triblock-copolymer (EPE) molecules into the pores of polymer framework. Aqueous EPE copolymer molecule had a tendency to aggregate to form clusters gradually and precipitated from water when the temperature is above a cloudy point. By adding EPE molecules into the acrylamide (AM) monomer solution, the mixture can be fabricated as uniform and transparent hydrogel via controlled radical polymerization. The polyacrylamide hydrogel is produced with a switchable optical property when subjecting to temperature variation. Such reversible thermally responsive material can be utilized as a functional material for smart window application. Additionally, the thermal responsive hydrogel is an inexpensive material, which is readily applicable as smart windows with significant reduction in material cost.
\end{abstract}

\section{Introduction}

Dynamic switchable window, also well known as "smart window," can adjust the amount of transmitted radiation in visible and infrared spectrum in response to the external stimuli [1-3]. Large-scale application of these smart windows may be highly desirable as it can drastically reduce the energy consumption of fully glazed building walls through reducing the demand for indoor loads of cooling-heating and electric lighting [4-7]. Various fabrication techniques have been developed for switchable windows for the past years [810]. Depending on the types of external stimuli, these smart devices can be divided into five categories: electrochromic, gasochromic, photochromic, thermochromic, and mechanical chromic [11-14]. Liquid crystal (LC) is a typical electrochromic material [15]. By applying an electric field between two conductive electrodes, LC molecules change their polarization in milliseconds, resulting in display transmittance from being transparent to opaque [16]. However, LC is relatively expensive and difficult to be fixed up [17]. Gasochromic windows have been developed based on the chromic properties of $\mathrm{WO}_{3}$ and noble metal doped $\mathrm{WO}_{3}$ [18-20].
Due to its high redox potential and sensitivity, $\mathrm{WO}_{3}$ can be colored easily by reacting with reductant gas, such as $\mathrm{H}_{2}$. But gasochromic devices require well-controlled exchange processes, whose potentially practical application is limited by short cycle lifetime [21]. Thermochromic windows are temperature sensitive. The commercialized "Suntuitive" manufactured by Pleotint is a thermochromic switchable sheet and can be cycled for thousands of times. On the other hand, inorganic smart window is commonly composed of vanadium dioxide $\left(\mathrm{VO}_{2}\right)$, which changes its phase at a critical temperature. Although transition metal oxides are durable, they will increase the material cost of smart window. More importantly, the low efficiency of simple transition metal oxides is a vital drawback for industrial products fabrication [22]. Photochromic and mechanical chromic materials are being investigated extensively in many applications [23]. Driven by the demand in cost reduction for smart windows, a new type of thermochromic smart window has been developed based on phase separation of triblock copolymer in aqueous state. The triblock-copolymer (EPE) molecules can be dispersed uniformly in water and turn to be a transparent solution. When the temperature is above critical temperature $\left(T_{c}\right)$ or 
cloudy point, the EPE molecules detach from water and aggregate to form clusters, resulting in solution from being transparent to opaque. This phase separation process is reversible and can be cycled for thousands of times, which contributes to hydrogel approach to be most probable application as smart window.

Herein, we present a novel thermally responsive hydrogel (TRH) by adding EPE into common polyacrylamide hydrogel and have successfully demonstrated its application as a smart window. By investigating the size variation of EPE molecules at different temperature and regulating the porosity of polyacrylamide (PAM) hydrogel, the optimized gelation and working conditions have been found. In this state, the transmittance of TRH can reach almost zero when the temperature is above $30^{\circ} \mathrm{C}$. It is found that the TRH based smart window can complete a phase separation process in $1 \mathrm{~min}$ using a $12 \mathrm{~V}$ DC power source or in 4 mins using a $9 \mathrm{~V}$ battery.

\section{Experimental Section}

2.1. Materials and Instruments. Acrylamide (AM), N,N-methylenebisacrylamide (Bis), ammonium persulfate (photoinitiator), $\mathrm{N}, \mathrm{N}, \mathrm{N}^{\prime}, \mathrm{N}^{\prime}$-tetramethylethylenediamine (TEMED), and poly(ethylene oxide), poly(propylene oxide), and poly(ethylene oxide) triblock copolymer (EPE) were purchased from Sigma-Aldrich. ITO glass was purchased from CSG of Shenzhen Display Technology Co. Ltd. The thickness of conductive layer is about $180 \mathrm{~nm}$. Sylgard 184 PDMS base and curing agent were obtained from Dow Corning (Midland, MI). ZetaPlus Zeta Potential Analyzer was from Brookhaven Instruments Corporation. Scanning Electronic Microscopy S-3000N was from Hitachi. UV-VIS spectrophotometer was Lambda 20 from Perkin Elmer. Topward Dual-Tracking DC Power supply 6303D was used in this study. LABCNCO D-090011 freezing drier was from South East Chemicals and Instruments Ltd.

2.2. PAM Hydrogel Preparation with Different Concentration. Acrylamide (AM) and N, $\mathrm{N}^{\prime}$-methylenebisacrylamide (Bis) $(19: 1, \mathrm{w} / \mathrm{w})$ were dissolved in water to form the monomer solution. The final concentrations of AM in monomer solution prepared were $5 \%, 10 \%, 15 \%$, and $20 \%$ (w/v), respectively. $0.5 \mathrm{~g} / \mathrm{mL}$ photoinitiator solution (PI solution) was prepared by dissolving $1.0 \mathrm{~g}$ ammonium persulfate in $2 \mathrm{~mL}$ DI water. Two $\mu \mathrm{L}$ TEMED and $2 \mathrm{~mL}$ monomer solution were mixed together. Then, the mixture was kept in the dark for few minutes via self-assembly where the solution solidified into hydrogel. The hydrogel was cleaned by DI water twice and placed in the refrigerator for 30 mins. Hydrogel then went through dehydration in a freeze-drier before subjecting to SEM imaging.

2.3. Dynamic Light Scattering Measurements of EPE Aqueous Solution. $1.0 \%(\mathrm{w} / \mathrm{v})$ EPE water solution was formed by dissolving $0.1 \mathrm{~g}$ EPE molecules in $10 \mathrm{~mL}$ DI water via selfassembly method. Then, $1 \mathrm{~mL} 1.0 \%$ (w/v) EPE water solution was pipetted and added to a plastic disposable cuvette for measurement.

2.4. Thermal Responsive Hydrogel (TRH) Preparation. $0.9 \%$ (w/v) TRH precursor solution was prepared by mixing $20 \mathrm{~mL}$ monomer solution, $20 \mu \mathrm{L}$ TEMED, $60 \mu \mathrm{L}$ PI solution, and $0.18 \mathrm{~g}$ EPE molecules and underwent ultrasonication for $1 \mathrm{~min}$. The mixture was kept in the dark for 10 mins for $\mathrm{TRH}$ formation via self-assembly method.

2.5. UV-VIS Spectrum Measurements of TRH at Different Temperature. $0.9 \%(\mathrm{w} / \mathrm{v}) \mathrm{TRH}$ precursor solution was pipetted into plastic cuvette. After complete gelation, the plastic cuvette was placed in water bath at a constant temperature and equilibrated for 5 mins. Then, the plastic cuvette was placed in the UV-VIS spectrophotometer and subjected to optical measurement between $400 \mathrm{~nm}$ and $1200 \mathrm{~nm}$. The recorded spectrum is specular transmittance without using integrating sphere. By varying the water bath temperature, we obtained a series of UV-VIS spectra for TRH at different temperatures $\left(20^{\circ} \mathrm{C}, 23^{\circ} \mathrm{C}, 26^{\circ} \mathrm{C}, 30^{\circ} \mathrm{C}\right.$, and $\left.40^{\circ} \mathrm{C}\right)$.

2.5.1. Passive Smart Window Fabrication. Two types of TRH based smart windows were fabricated. The passive smart window was formed by bonding four thin PDMS stripes between two glass plates of $10 \mathrm{~cm} * 10 \mathrm{~cm}$, where the PDMS stripes define the four edges of the smart window while the two glass plates define the top and bottom boundaries. The small gaps between PDMS stripes and glass plates were sealed off using wax, except for a single opening that is large enough for a syringe needle of about $0.9 \mathrm{~mm}$ in diameter. The enclosed space was filled with $0.9 \%$ (w/v) TRH precursor solution using a syringe via the opening. It was then placed on a steel plate to absorb the heat generated during polymerization $[24,25]$. Once polymerization was completed, the opening was sealed off by wax, forming a passive smart window.

2.5.2. Active Smart Window Fabrication. ITO electrodes on glass plates of $10 \mathrm{~cm} \times 10 \mathrm{~cm}$ were patterned by standard lithography processes: (1) ITO coated glass plates were cleaned by acetone (one time), ethanol (two times), and water (three times); (2) a layer of 504 photoresist was spin-coated onto ITO glass plates $(3000 \mathrm{r} / \mathrm{s}, 30 \mathrm{~s})$ and baked for $1 \mathrm{~min}$ on a hot plate at $110^{\circ} \mathrm{C}$; (3) the photoresist was exposed to UV for $7 \mathrm{~s}$ and the patterns was developed using FHD-5 solution; (4) the glass plates were wet-etched by a standard etching solution for $8 \mathrm{mins}$ followed by a lift-off process; (5) the glass plates with patterned ITO electrodes were postbaked for 5 mins.

A layer of PDMS with weight ratio of monomer and curing agent of $10: 1$ was spin-coated onto the patterned glass plates at $2000 \mathrm{r} / \mathrm{s}$ for $60 \mathrm{~s}$. Then, the plates were baked at $80^{\circ} \mathrm{C}$ for 30 mins for PDMS solidification. The PDMS layer is used for preventing electrolysis at ITO electrodes. The ITO plates with PDMS insulating layers were then subjected to the same fabrication process for passive smart window above (Figure 5(a)). 


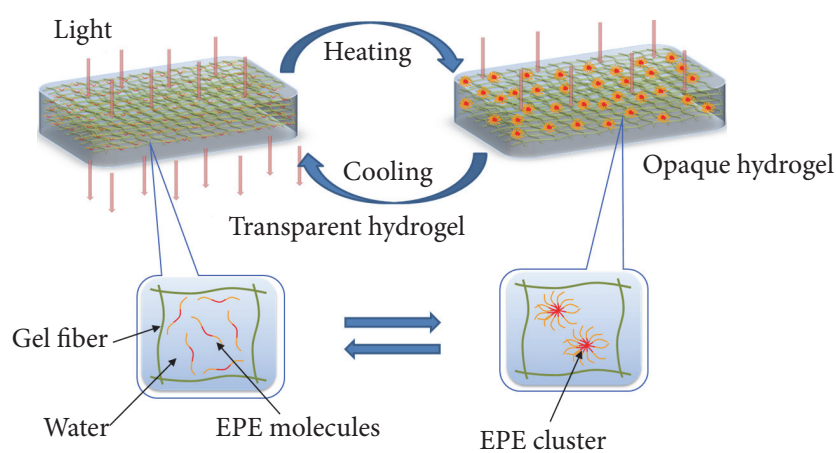

Figure 1: A schematic illustration of the thermal response mechanism of the hydrogel. At about $20^{\circ} \mathrm{C}$, EPE molecules disperse in water uniformly among TRH. At initial stage, the TRH is transparent to naked eyes. When the temperature reaches the critical temperature, EPE molecules aggregate to form clusters (orange dots). The EPE clusters then scatter visible light and hydrogel appears as opaque. The phase separation process is reversible after the temperature drops below the critical value.

\section{Results and Discussions}

3.1. Mechanism of Smart Window. Individual EPE molecule is composed of three blocks: poly(ethylene oxide) (PEO), poly(propylene oxide) (PPO), and poly(ethylene oxide) (PEO), well known as a triblock copolymer. The micelle formation mechanism of EPE in water has been investigated extensively for many years [26]. The core of the micelles is composed of hydrophobic PPO blocks, which are surrounded by hydrophilic PEO chains. In brief, EPE molecules can be self-assembled to form micelles in aqueous solution above the critical micellization temperature owing to the dehydration of PPO block. When the temperature reaches the critical value, the micelles aggregate and form clusters through entangling between hydrophilic coronal PEO chains. In this case, the EPE molecules are no longer soluble and precipitate as another phase. In other words, the solution turns to be haze or cloudy when the temperature is above the cloudy point. Meanwhile, EPE solution turns transparent again when the temperature is lower than $T_{c}$. The transparency-opaque switchable effect can be reversible as shown in Figure 1.

The size distribution of EPE molecules was determined via dynamic light scattering in aqueous solution, which might have an influence on the pore size of framework. Take the sample with $1 \%(\mathrm{w} / \mathrm{v})$ EPE solution, for example, cluster size distribution of EPE molecules was investigated at four different temperatures: $20^{\circ} \mathrm{C}, 25^{\circ} \mathrm{C}, 30^{\circ} \mathrm{C}$, and $40^{\circ} \mathrm{C}$. At $20^{\circ} \mathrm{C}$, the particles size was under 4 nanometers and they were observed (Figure 2(a)). At $25^{\circ} \mathrm{C}$, EPE molecules started to aggregate and form clusters with average size of about 1200 nanometers (Figure 2(b)). At this time, the EPE clusters were starting to form in a loosely packed situation, which results in a relatively large cluster size with low cluster amounts. In the vision of sample, the EPE aqueous solution gradually turned to be haze between transparent and opaque. Further, when the temperature reached above $30^{\circ} \mathrm{C}$, the EPE molecules completed the aggregation processing, which causes the uniform and highly packed clusters to be apparent (Figures 2(c) and 2(d)). In this process, the EPE aqueous solution changed from being clear to cloudy. Since EPE molecules are far smaller than individual pore size of the PAM hydrogel, the behavior of EPE molecules in hydrogel will not be affected by the hydrogel fibers.

In this study, polyacrylamide network was utilized to confine separated small amount of EPE molecules in solution. Each EPE molecule will distribute uniformly in bulk hydrogel. In a microscopic view, the hydrogel is a fiber-like net made of polymer and aqueous solution. The aqueous solution fills up the pores formed by cross-linked fibers. Since EPE molecules are far smaller than the size of the pores, the behavior of EPE molecules is similar to them in bulk aqueous solution even though they are confined within a single pore. The fiber-like structure of the hydrogel behaved like a reservoir for $\mathrm{H}_{2} \mathrm{O}$ molecules. The entire phase separation process for EPE molecules within PAM hydrogel should be the same as that occurring in bulk aqueous solution, as illustrated in Figure 1 . The new thermal response property of PAM hydrogel can be achieved only by adding EPE molecules before polymerization process, without needing complex process and treatment, which is very useful for end applications.

\subsection{Pore Size and Porosity Regulation of PAM Hydrogel. Since} the size distribution of EPE clusters was determined, the porosity of hydrogel was needed to be investigated. Hydrogel has been widely used in surface chemistry, separation science, and tissue engineering [27]. Fabricated PAM hydrogel was composed of cross-linked polymer fiber grids which can hold up a large amount of $\mathrm{H}_{2} \mathrm{O}$ molecules. Hydrogel has been widely studied as separation matrix in DNA gel electrophoresis and size exclusion chromatography $[28,29]$. Thus, pore size and porosity distribution of PAM hydrogel can be regulated easily. Compared with other hydrogels extracted from natural products, such as alginate and agarose, PAM hydrogel is stable, reproducible, and cheap [30]. Moreover, the polymerization process of AM monomer solution to form the hydrogel was not affected by the EPE, making PAM hydrogel a suitable carrier for EPE molecules.

The pore size and porosity of hydrogel had a strong effect on the behavior of EPE molecules. In this work, the porosity was regulated by varying the concentration of AM monomer in precursor solution and the degree of cross-linking. We prepared four kinds of precursor solution with 5\% (w/v), 10\% $(\mathrm{w} / \mathrm{v}), 15 \%(\mathrm{w} / \mathrm{v})$, and 20\% (w/v) AM monomer, respectively. The weight ratio of AM monomer and Bis (cross-linker) was fixed at 19:1. After polymerization and freeze-drying, the skeleton of polymer fibers (dehydrated hydrogel) was characterized by SEM as shown in Figure 3. It was obvious that the pore size remained around 10 micrometers for different AM monomer concentrations, and there was no significant change in the degree of porosity. The porosity enhanced the possibility of having EPE clusters trapped in polymer fiber during polymerization, affecting the degree of phase transition within hydrogel. As shown in Figures 3(a)-3(d), it was found that hydrogel prepared from $5 \%(w / v)$ to $20 \%(\mathrm{w} / \mathrm{v})$ AM monomer solution is suitable for our smart window experiments. Furthermore, the AM monomer concentration was fixed at $5 \%$ with the weight ratios of AM monomer 


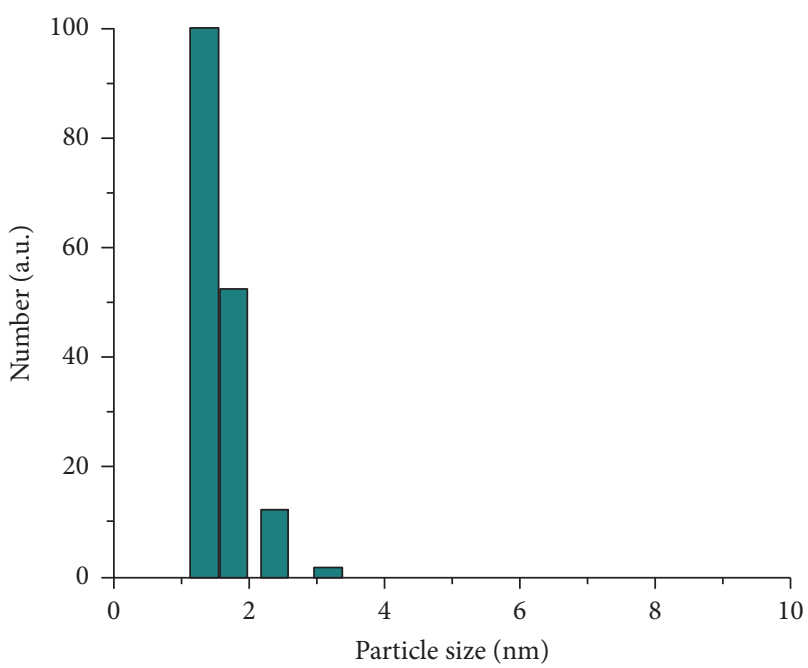

(a)

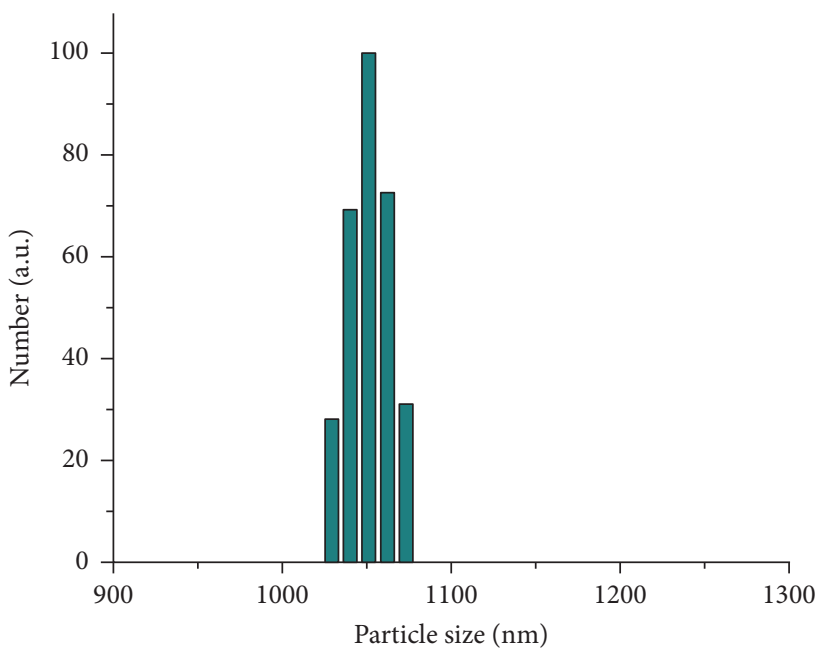

(c)

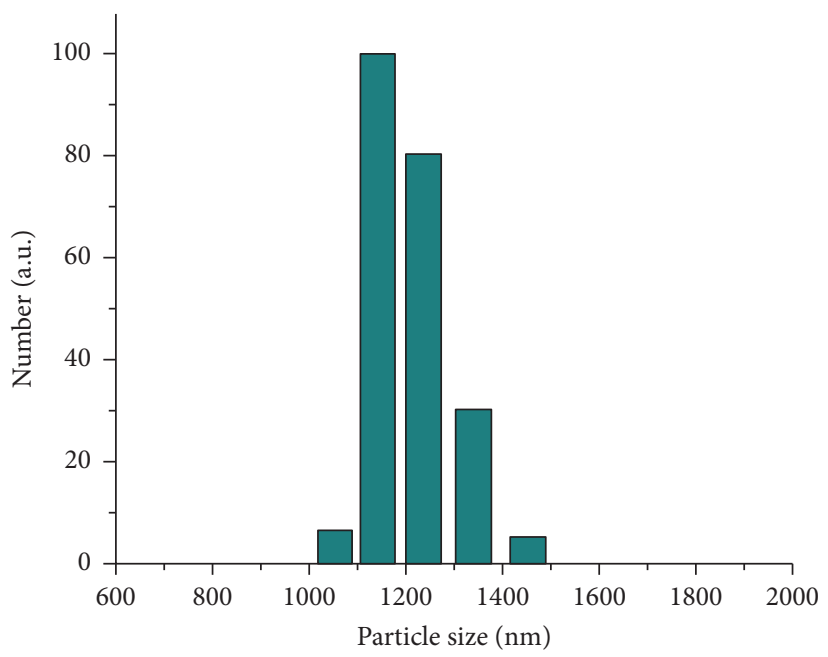

(b)

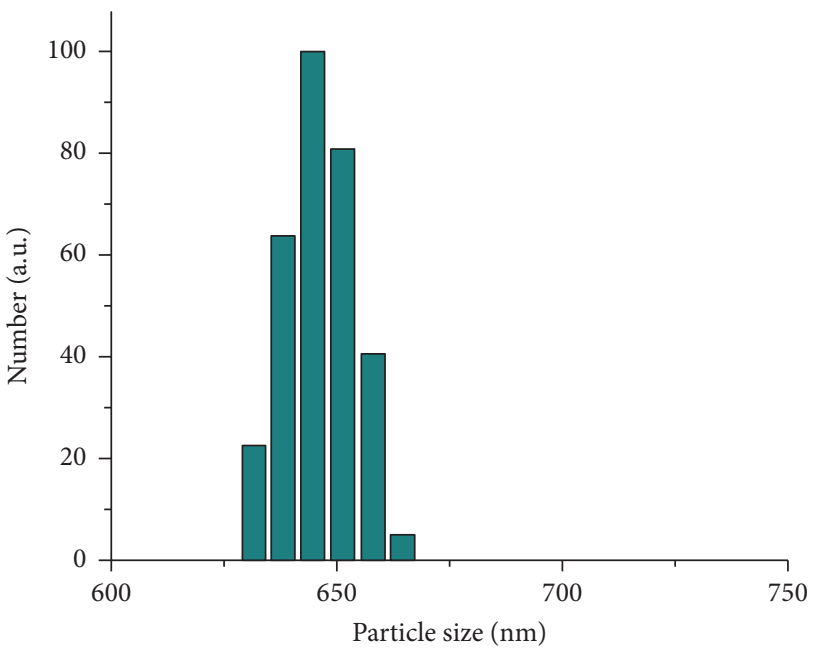

(d)

Figure 2: The size distribution of EPE molecule clusters (1\% wt EPE water solution) at different temperatures: (a) $20^{\circ} \mathrm{C},(\mathrm{b}) 25^{\circ} \mathrm{C},(\mathrm{c}) 30^{\circ} \mathrm{C}$, and (d) $40^{\circ} \mathrm{C}$.

and Bis (cross-linker) of $19: 1,15: 1$, and $10: 1$, respectively. The PAM hydrogel prepared from precursor solution with 19:1 weight ratio was transparent, while others appeared to be translucent. Therefore, the cross-linking degree of polymer fibers affects the transmittance of hydrogel. For smart window fabrication, AM monomer concentration was used as $5 \%(\mathrm{w} / \mathrm{v})$ with a weight ratio of Bis at 1:19.

3.3. Applications Studies of TRH in Smart Window. In the framework of PAM hydrogel, a specific amount of EPE molecules was added to the precursor solution to form TRH to implement the thermal response in PAM hydrogel. The concentration of EPE molecules affected the degree of transparency and its phase separation process. Firstly, three kinds of TRH were prepared with different concentrations of EPE molecules: 0.8\% (w/v), 1.0\% (w/v), and $1.2 \%(\mathrm{w} / \mathrm{v})$, respectively. For $0.8 \%(\mathrm{w} / \mathrm{v})$ EPE in TRH, the degree of transparency of TRH appeared to be satisfactory by naked eyes. However, it cannot achieve complete opaqueness above the critical temperature, because the concentration of EPE molecules was too low to form enough clusters to achieve visible opaqueness. By increasing the EPE concentration to $1.0 \%(\mathrm{w} / \mathrm{v})$ and $1.2 \%(\mathrm{w} / \mathrm{v})$, TRH can turn entirely opaque after reaching the critical temperature. However, the initial state of TRH showed uneven cloudiness observed by naked eyes. The unevenness became more severe in higher concentration of EPE sample. Therefore, the concentration of EPE molecules was optimized at $0.9 \%(\mathrm{w} / \mathrm{v})$ for a sharp optical transition between transparency and opaqueness.

The UV-visible transmission spectrum of TRH made from 5\%(w/v) AM monomer and 0.9\% (w/v) EPE was measured as shown in Figure 4 . At $20^{\circ} \mathrm{C}$, the transmittance of TRH was comparable with pure DI water. As the temperature increased, the transmittance of TRH reduced gradually (Figure $4(\mathrm{a})$ ). When the temperature was above $30^{\circ} \mathrm{C}$, as 


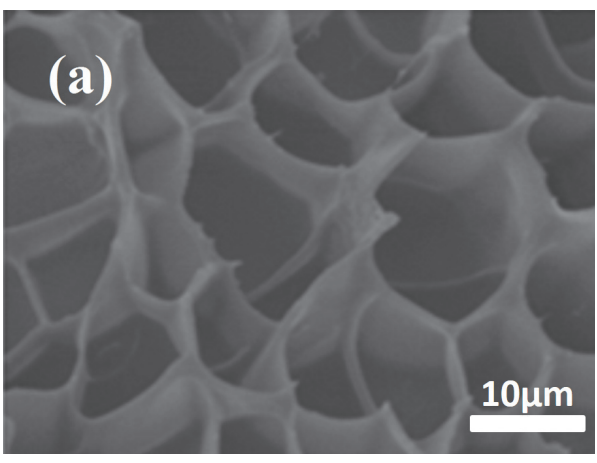

(a)

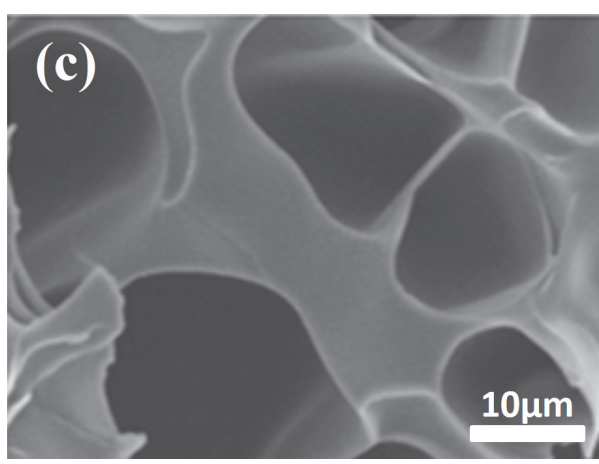

(c)

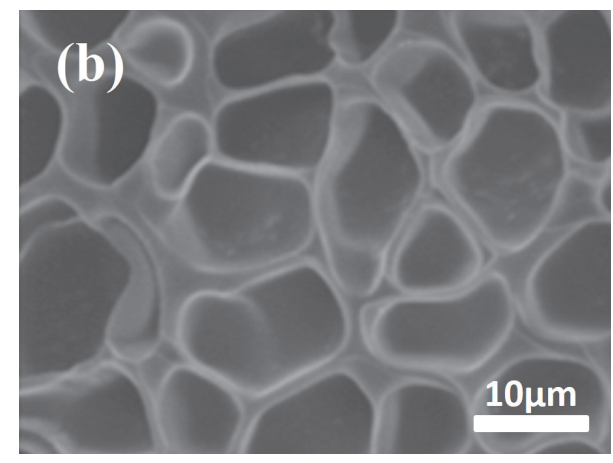

(b)

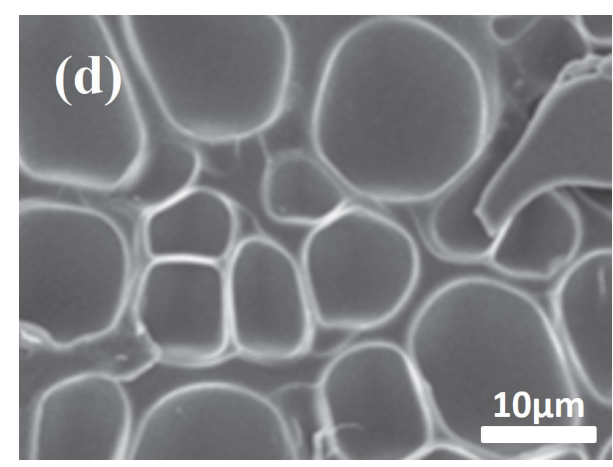

(d)

FIGURE 3: SEM images of polyacrylamide gel with different concentrations: (a) 5\% (w/v) acrylamide, (b) 10\%, (c) 15\%, and (d) $20 \%$.

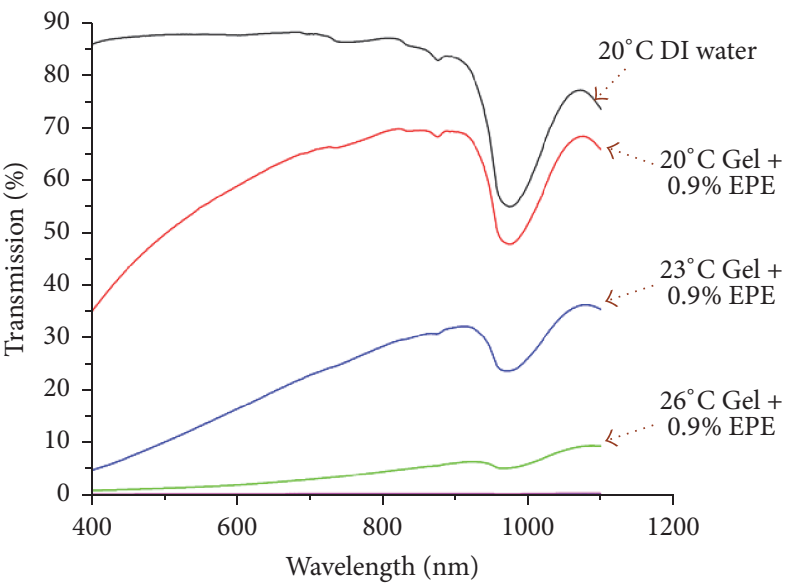

(a)

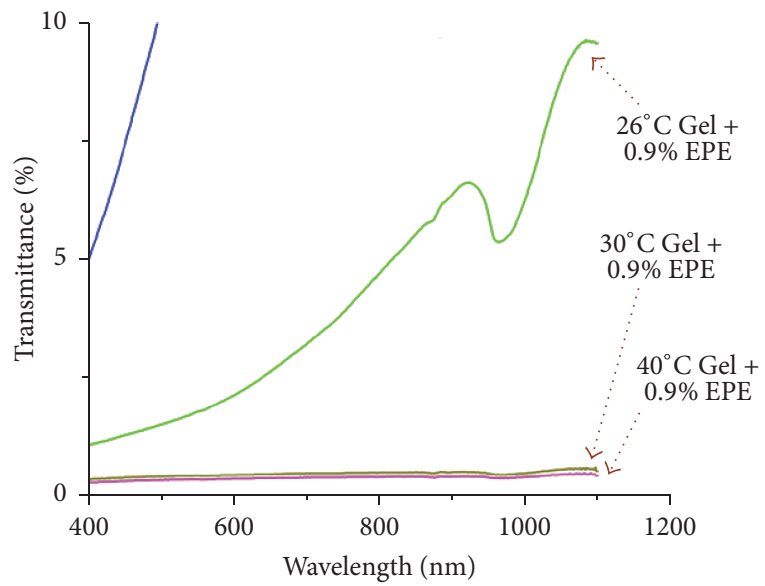

(b)

FIgURE 4: The UV-VIS transmission spectra of $5 \%(\mathrm{w} / \mathrm{v})$ polyacrylamide gel containing $0.9 \%(\mathrm{w} / \mathrm{v})$ EPE molecules at different temperatures. (a) Transmission spectra of DI water and TRH at different temperatures (from $20^{\circ} \mathrm{C}$ to $40^{\circ} \mathrm{C}$ ). (b) The local magnification of the transmission spectra for TRH at $30^{\circ} \mathrm{C}$ and $40^{\circ} \mathrm{C}$.

shown in Figure 4(b), TRH appeared to be cloudy and the transmittance approaches zero.

From the experimental results, the obvious application for the optimized TRH is as dynamic switchable window or smart window owing to its capability of changing from transparency to opaqueness by adjusting external temperature. The phase separation process of TRH is reversible and can be cycled for many times. In this study, two types of smart window had been successfully fabricated based on TRH: one was passive smart window and the other was active smart window.

The passive smart window was fabricated by sandwiching a layer of TRH between two ordinary glass plates. The smart window changed from being transparent to opaque in response to temperature variation from the surrounding environment. Such smart windows can be fabricated in 


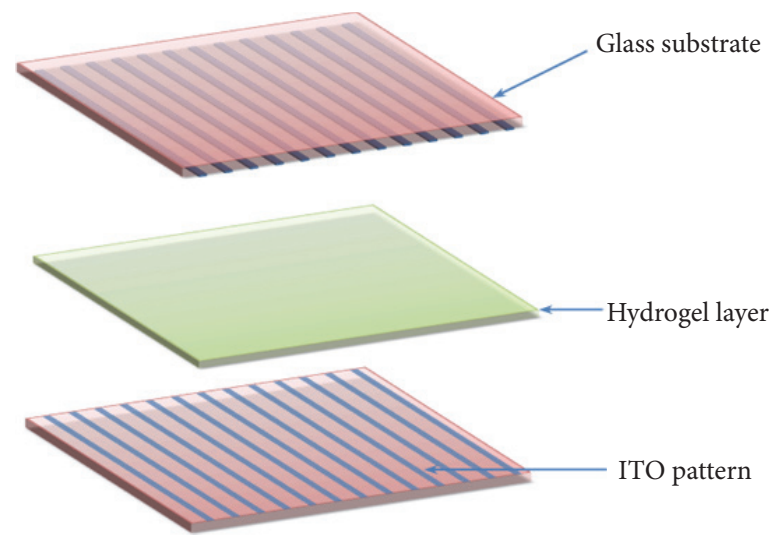

(a)
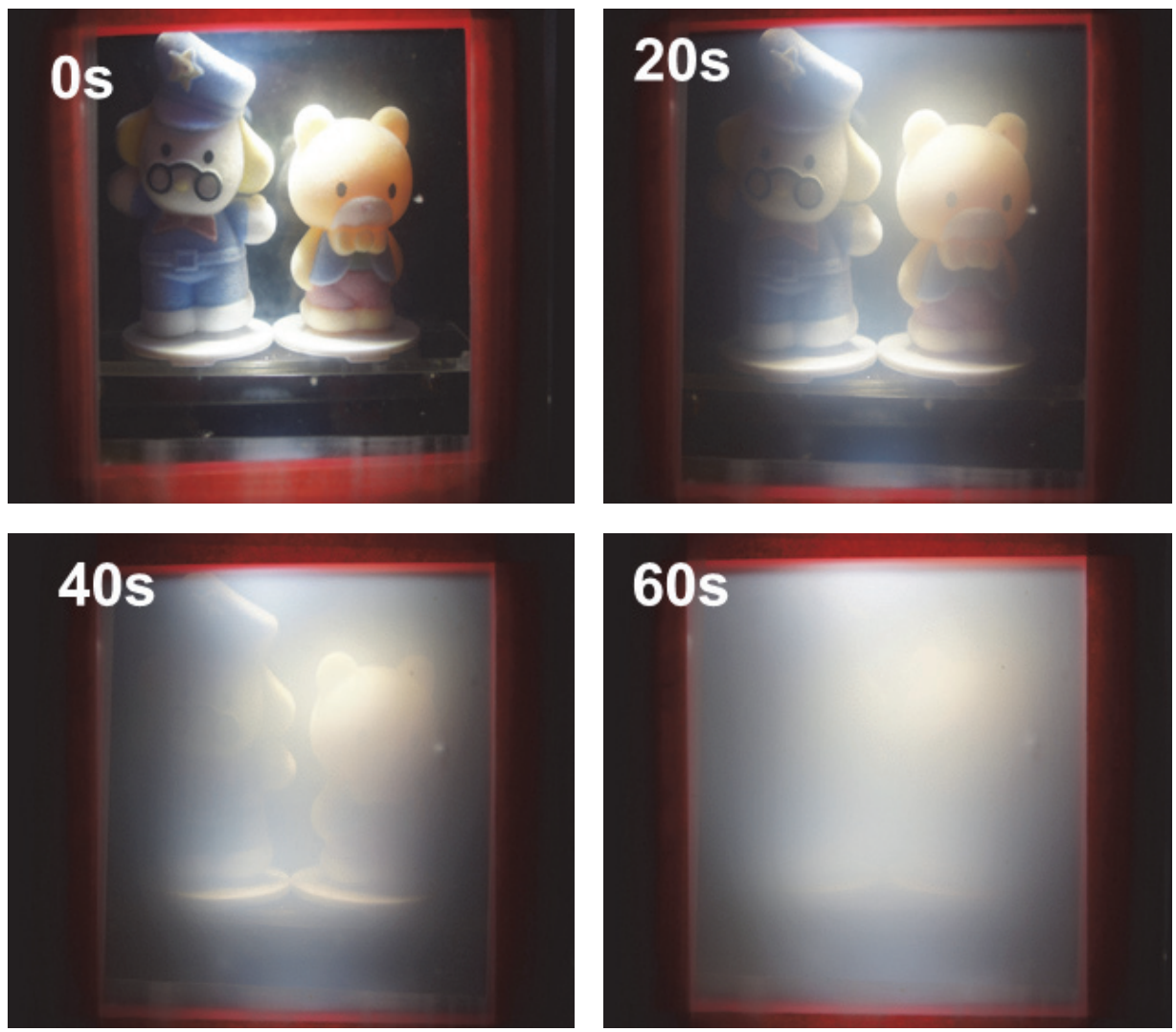

(b)

FIGURE 5: The phase separation process of active smart window. (a) Smart window is composed of 3 layers: top and bottom layers are glass plates with patterned ITO electrodes; middle layer is made of thermally responsive hydrogel (TRH). (b) Smart window changes from being transparent to opaque in 1 min under $12 \mathrm{~V} \mathrm{DC}$.

a large-scale type as decoration or covering materials for building walls. When the external temperature is higher than the critical value, the smart window changes from transparency to opaqueness. The TRH based smart windows could block incident sunlight to protect our privacy.

The active smart window was composed of a middle layer made of TRH and sandwiched by two glass plates with patterned ITO electrodes (Figure 5(a)). Under an external electric field, ITO electrodes could lead the TRH layer phase separation. In our demonstration, the active smart window was connected with a $12 \mathrm{~V}$ DC power supply, which took about $1 \mathrm{~min}$ for optical change (Figure 5(b)). In other words, the phase separation process of EPE molecules was completely achieved in $1 \mathrm{~min}$. Sample experimental procedure was performed via using a $9 \mathrm{~V}$ battery (Figure 6). It took about 4 mins for the smart window to turn opaque. The speed of the phase transition was highly influenced by the amount of current drained from the battery. By supplying an external 


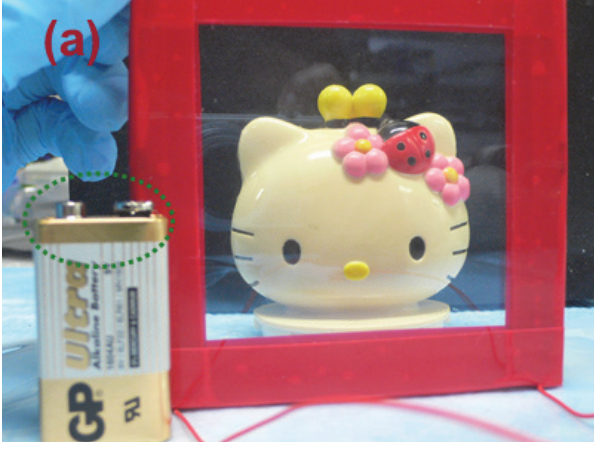

(a)

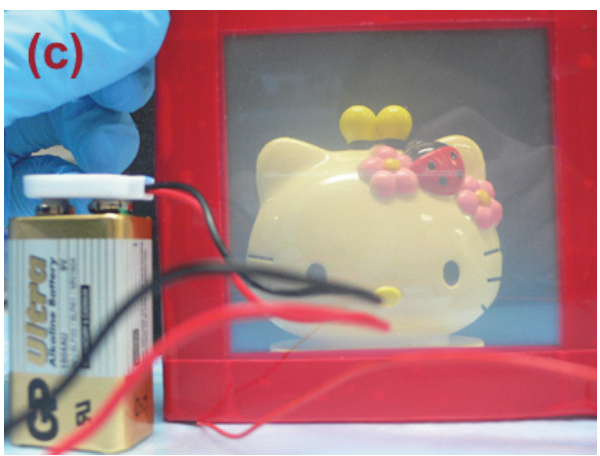

(c)

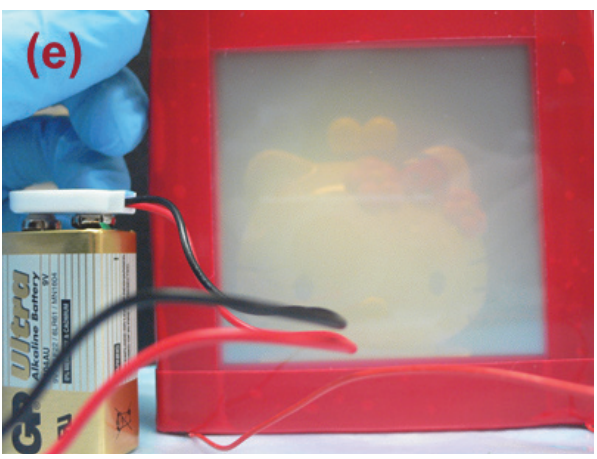

(e)

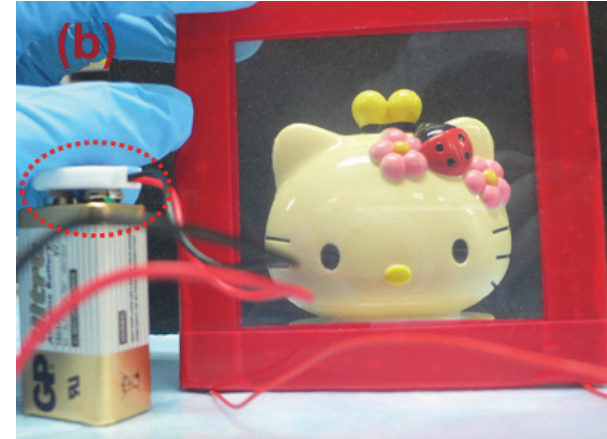

(b)

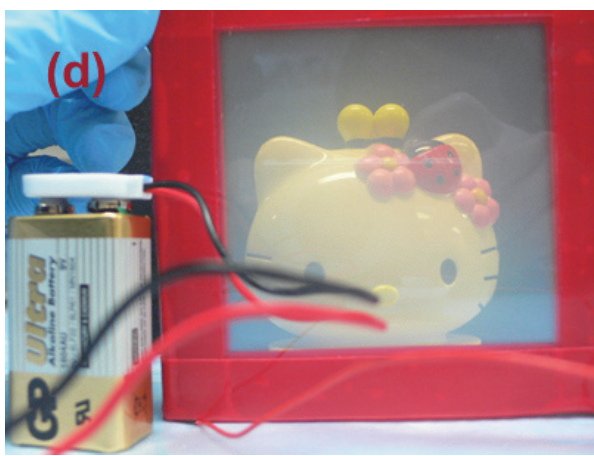

(d)

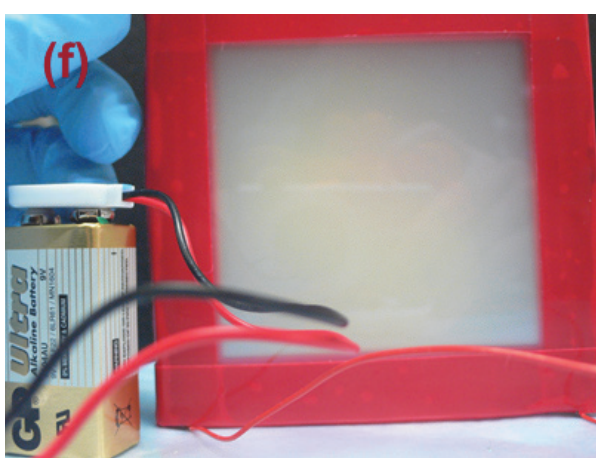

(f)

Figure 6: The phase separation process of active smart window using a $9 \mathrm{~V}$ battery. (a) No external power supply. $9 \mathrm{~V}$ battery is disconnected. (b) Zero min after electrical connection. (c) One min. (d) Two mins. (e) Three mins. (f) Four mins.

power source, the active smart window can now be used or installed and is not affected by external environmental conditions such as humidity, sunlight, location (indoor or outdoor), and temperature.

Comparing with other thermodynamic smart windows, TRH based smart window offers an obvious advantage in material cost. PAM hydrogel is a common and readily available material in chemical industry and the raw material cost for fabricating hydrogel is low. The material cost for fabricating TRH with a dimension of $1 \mathrm{~m} * 1 \mathrm{~m} * 3 \mathrm{~mm}$ (length $*$ width $*$ height) is less than USD 0.65 , while, the material cost for a one square meter of passive smart window is only 100 cents USD more expensive than a plain glass sheet of the same size. Large-scale production of these smart windows can drastically reduce the unit cost per square meter, as wallcovering material for green houses, highly glazed buildings, interior design, and decoration.

\section{Conclusion}

In summary, a thermally responsive hydrogel was presented by confining triblock copolymer (EPE) within the pores of the polyacrylamide gel networks (PAM hydrogel). The TRH can undergo phase separation induced by heat effect, leading to the change in its optical property (from transparent to opaque) above the critical temperature. When the temperature is lower than critical point, hydrogel will turn back to be transparent again. Due to this reversible thermal 
response, TRH was used as a low-cost material for smart window application. Two types of TRH based smart windows (active and passive) have been demonstrated. The induction time can be several minutes when applying external power supply, and a small amount of energy is consumed during the process. Since PAM hydrogel is common and readily abundant in chemical industry, large-scale production of TRH based smart window is feasible. More interestingly, there are many more interesting applications for TRH smart windows in daily life when privacy is required.

\section{Competing Interests}

The authors declare that there are no competing interests regarding the publication of this manuscript.

\section{References}

[1] R. Baetens, B. P. Jelle, and A. Gustavsen, "Properties, requirements and possibilities of smart windows for dynamic daylight and solar energy control in buildings: a state-of-the-art review," Solar Energy Materials and Solar Cells, vol. 94, no. 2, pp. 87-105, 2010.

[2] Y. Wang, E. L. Runnerstrom, and D. J. Milliron, "Switchable Materials for Smart Windows," Annual Review of Chemical and Biomolecular Engineering, vol. 7, no. 1, pp. 283-304, 2016.

[3] S.-M. Wang, L. Liu, W.-L. Chen, and E.-B. Wang, "High performance visible and near-infrared region electrochromic smart windows based on the different structures of polyoxometalates," Electrochimica Acta, vol. 113, pp. 240-247, 2013.

[4] C. G. Granqvist, A. Azens, A. Hjelm et al., "Recent advances in electrochromics for smart windows applications," Solar Energy, vol. 63, no. 4, pp. 199-216, 1998.

[5] J. S. E. M. Svensson and C. G. Granqvist, "Electrochromic hydrated nickel oxide coatings for energy efficient windows: optical properties and coloration mechanism," Applied Physics Letters, vol. 49, no. 23, pp. 1566-1568, 1986.

[6] A. Hauch, A. Georg, S. Baumgärtner, U. O. Krašovec, and B. Orel, "New photoelectrochromic device," Electrochimica Acta, vol. 46, no. 13-14, pp. 2131-2136, 2001.

[7] L.-M. Huang, C.-W. Hu, H.-C. Liu, C.-Y. Hsu, C.-H. Chen, and K.-C. Ho, "Photovoltaic electrochromic device for solar cell module and self-powered smart glass applications," Solar Energy Materials and Solar Cells, vol. 99, pp. 154-159, 2012.

[8] G. Gunbas and L. Toppare, "Electrochromic conjugated polyheterocycles and derivatives-highlights from the last decade towards realization of long lived aspirations," Chemical Communications, vol. 48, no. 8, pp. 1083-1101, 2012.

[9] B. P. Jelle, A. Hynd, A. Gustavsen, D. Arasteh, H. Goudey, and R. Hart, "Fenestration of today and tomorrow: a state-of-the-art review and future research opportunities," Solar Energy Materials and Solar Cells, vol. 96, no. 1, pp. 1-28, 2012.

[10] J. Zhou, Y. Gao, Z. Zhang et al., " $\mathrm{VO}_{2}$ thermochromic smart window for energy savings and generation," Scientific Reports, vol. 3, article 3029, 2013.

[11] R. Pardo, M. Zayat, and D. Levy, "Photochromic organic-inorganic hybrid materials," Chemical Society Reviews, vol. 40, no. 2, pp. 672-687, 2011.

[12] G. Teowee, T. Gudgel, K. McCarthy, A. Agrawal, P. Allemand, and J. Cronin, "User controllable photochromic (UCPC) devices," Electrochimica Acta, vol. 44, no. 18, pp. 3017-3026, 1999.

[13] F.-H. Huang, C.-C. Chen, D.-J. Lin, T.-M. Don, and L.-P. Cheng, "Effect of particle size on the photochromic response of PWA/ $\mathrm{SiO}_{2}$ nanocomposite," Journal of Nanoparticle Research, vol. 12, no. 8, pp. 2941-2950, 2010.

[14] A. Seeboth, R. Ruhmann, and O. Mühling, "Thermotropic and thermochromic polymer based materials for adaptive solar control," Materials, vol. 3, no. 12, pp. 5143-5168, 2010.

[15] S. G. Lee, D. Y. Lee, H. S. Lim, D. H. Lee, S. Lee, and K. Cho, "Switchable transparency and wetting of elastomeric smart windows," Advanced Materials, vol. 22, no. 44, pp. 5013-5017, 2010.

[16] J. Y. Luo, S. Z. Deng, Y. T. Tao et al., "Evidence of localized water molecules and their role in the gasochromic effect of $\mathrm{WO}_{3}$ nanowire films," Journal of Physical Chemistry C, vol. 113, no. 36, pp. 15877-15881, 2009.

[17] K. Yoshimura, Y. Yamada, S. Bao, K. Tajima, and M. Okada, "Preparation and characterization of gasochromic switchablemirror window with practical size," Solar Energy Materials and Solar Cells, vol. 93, no. 12, pp. 2138-2142, 2009.

[18] M. Ranjbar, N. T. Garavand, S. M. Mahdavi, and A. Iraji zad, "Electroless plating of palladium on $\mathrm{WO}_{3}$ films for gasochromic applications," Solar Energy Materials and Solar Cells, vol. 94, no. 2, pp. 201-206, 2010.

[19] M. Ranjbar, S. Fardindoost, S. M. Mahdavi, A. Iraji Zad, and N. Tahmasebi G, "Palladium nanoparticle deposition onto the $\mathrm{WO}_{3}$ surface through hydrogen reduction of $\mathrm{PdCl}_{2}$ : characterization and gasochromic properties," Solar Energy Materials and Solar Cells, vol. 95, no. 8, pp. 2335-2340, 2011.

[20] L. Kang, Y. Gao, H. Luo et al., "Thermochromic properties and low emissivity of $\mathrm{ZnO}: \mathrm{Al} / \mathrm{VO}_{2}$ double-layered films with a lowered phase transition temperature," Solar Energy Materials and Solar Cells, vol. 95, no. 12, pp. 3189-3194, 2011.

[21] D. Gogova, L.-K. Thomas, and B. Camin, "Comparative study of gasochromic and electrochromic effect in thermally evaporated tungsten oxide thin films," Thin Solid Films, vol. 517, no. 11, pp. 3326-3331, 2009.

[22] V. Shrotriya, G. Li, Y. Yao, C.-W. Chu, and Y. Yang, "Transition metal oxides as the buffer layer for polymer photovoltaic cells," Applied Physics Letters, vol. 88, no. 7, Article ID 073508, 2006.

[23] Y. El Sayed Said, "Linear and non-linear optical phenomena of glasses (photonics-photo chromic-electro and magneto optics): a review," Solid State Phenomena, vol. 207, pp. 1-35, 2014.

[24] J.-S. Wang and K. Matyjaszewski, "Controlled/'living' radical polymerization. Atom transfer radical polymerization in the presence of transition-metal complexes," Journal of the American Chemical Society, vol. 117, no. 20, pp. 5614-5615, 1995.

[25] K. Matyjaszewski, Controlled Radical Polymerization, American Chemical Society, 1998.

[26] P. Alexandridis, V. Athanassiou, and T. A. Hatton, "PluronicP105 PEO-PPO-PEO block copolymer in aqueous urea solutions: micelle formation, structure, and microenvironment," Langmuir, vol. 11, no. 7, pp. 2442-2450, 1995.

[27] A. Li, X. Chen, and V. M. Ugaz, "Miniaturized system for rapid field inversion gel electrophoresis of DNA with real-time wholegel detection," Analytical Chemistry, vol. 82, no. 5, pp. 1831-1837, 2010.

[28] F. Lv, P. Zhu, C. Wang, and L. Zheng, "Preparation, characterization, and dyeing properties of calcium alginate fibers," Journal of Applied Polymer Science, vol. 126, supplement 1, pp. E383-E388, 2012. 
[29] D. K. Yang, L. Shi, and R. Bao, "Bistable switchable liquid crystal window," US Patent 8,913,215, 2014.

[30] D. Cupelli, F. P. Nicoletta, S. Manfredi et al., "Self-adjusting smart windows based on polymer-dispersed liquid crystals," Solar Energy Materials and Solar Cells, vol. 93, no. 11, pp. 20082012, 2009. 

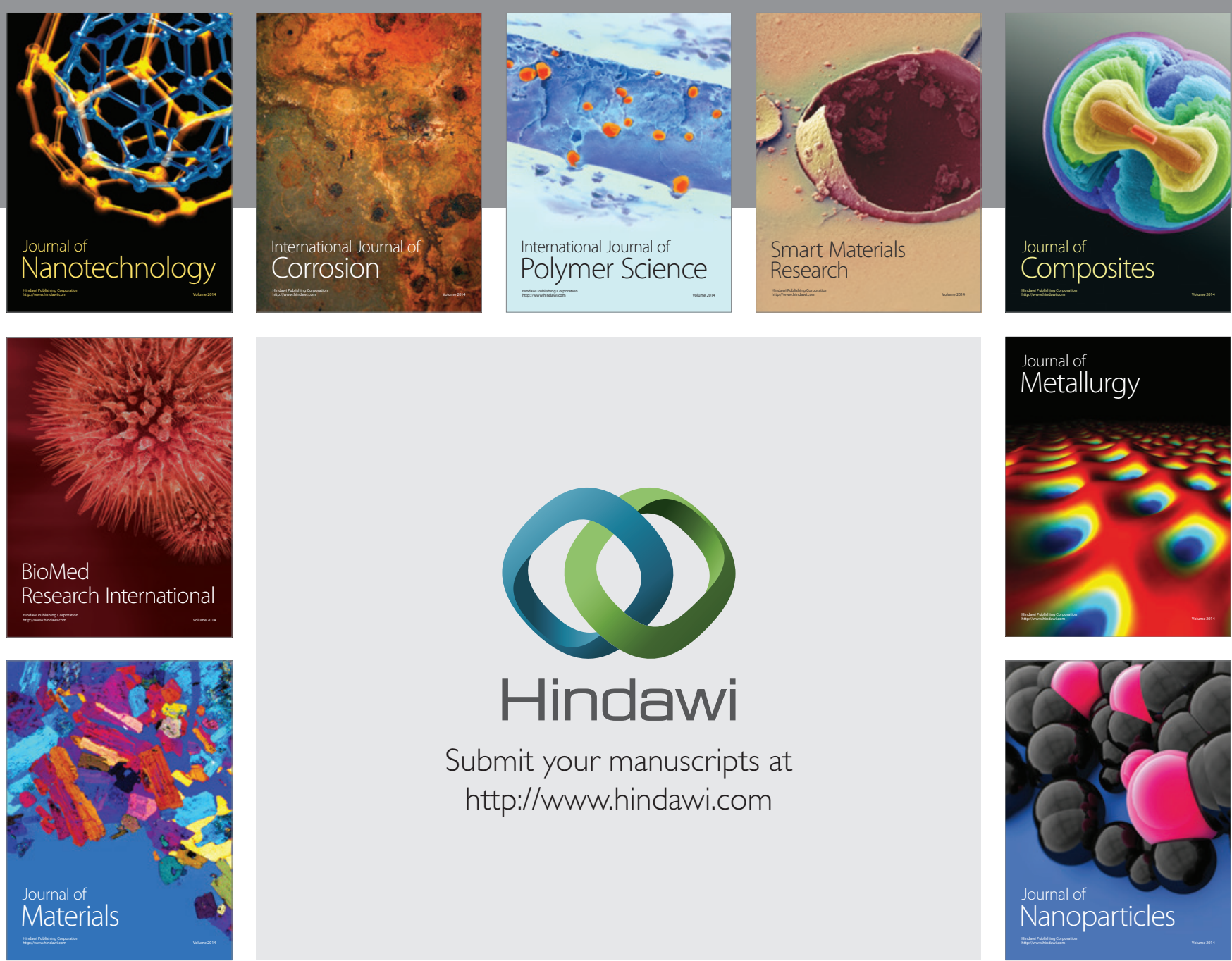

\section{Hindawi}

Submit your manuscripts at

http://www.hindawi.com

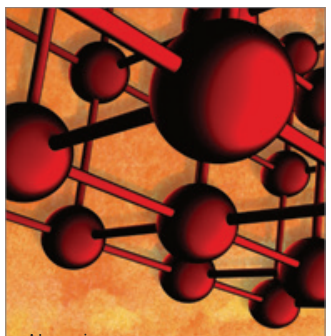

Materials Science and Engineering
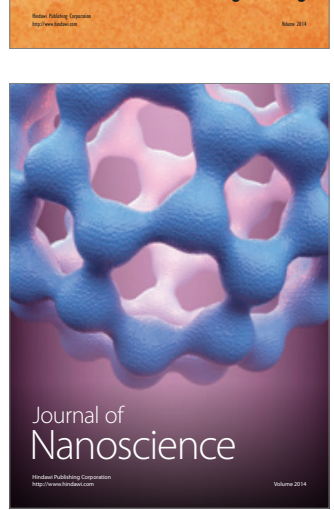
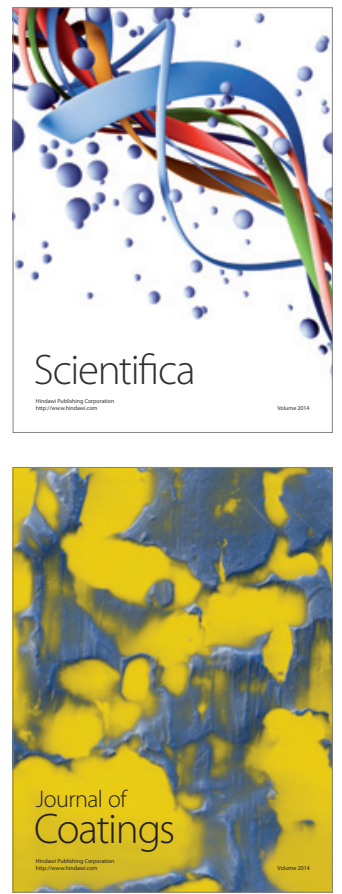
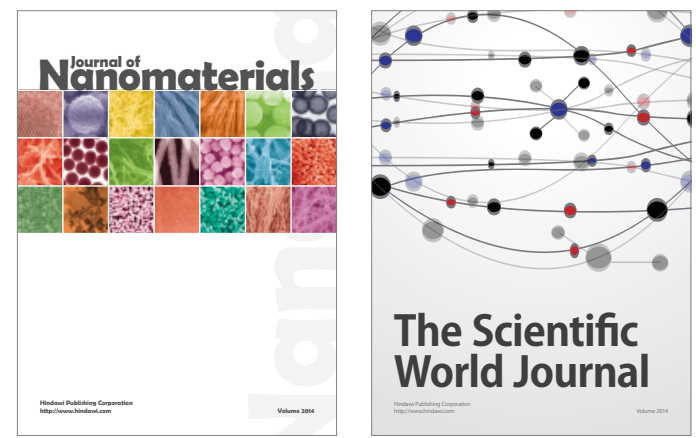

The Scientific World Journal
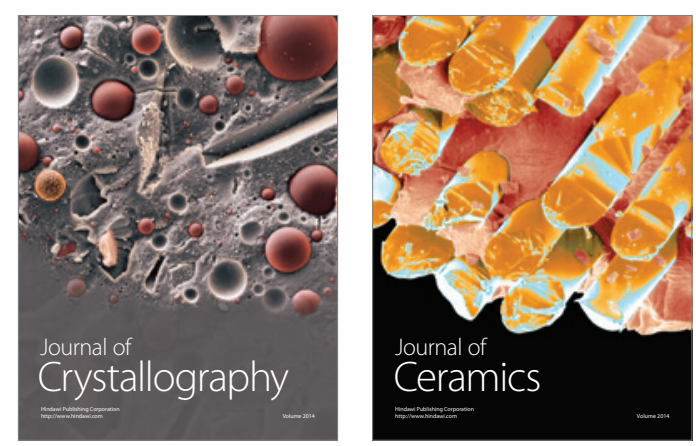
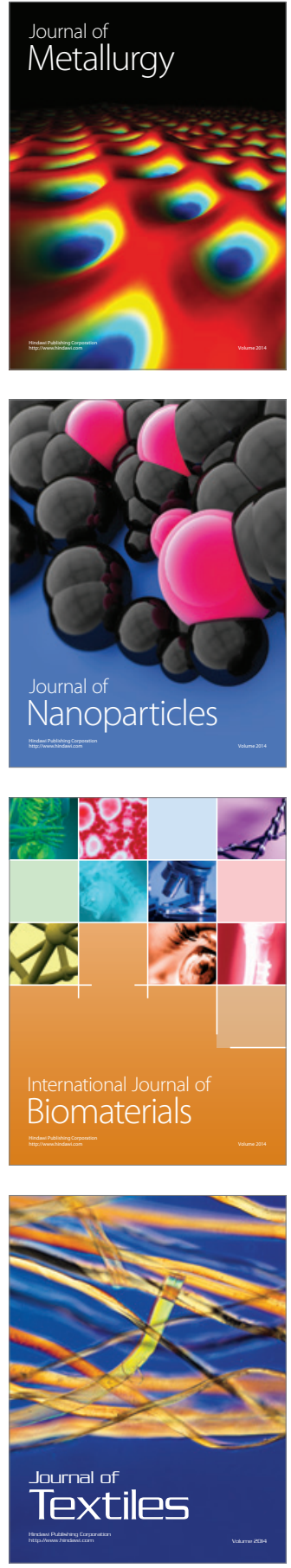